\title{
Helios Expressing Regulatory T Cells are Correlated with Decreased IL-2 Producing CD8 T Cells and Antibody Diversity in Mozambican Individuals Living Chronically HIV-1
}

Raquel Matavele Chissumba ( $\square$ raquelmatavele@gmail.com )

Instituto Nacional de Saúde

Cacildo Magul

Instituto Nacional de Saúde

Rosa Macamo

Instituto Nacional de Saúde

Vânia Monteiro

Instituto Nacional de Saúde

Maria Enosse

Instituto Nacional de Saúde

Ivalda Macicame

Instituto Nacional de Saúde

Victória Cumbane

Instituto Nacional de Saúde

Nilesh Bhatt

Instituto Nacional de Saúde

\section{Edna Viegas}

Instituto Nacional de Saúde

Michelle Imbach

U.S. Military HIV Research Program, Walter Reed Army Institute of Research

Leigh Anne Eller

U.S. Military HIV Research Program, Walter Reed Army Institute of Research

Christina S. Polyak

U.S. Military HIV Research Program, Walter Reed Army Institute of Research

Luc Kestens

Institute of Tropical Medicine Antwerp

llesh Jani

Instituto Nacional de Saúde 


\section{Research Article}

Keywords: Seronegativity, Tregs, HIV chronic infection, Helios

Posted Date: August 10th, 2021

DOl: https://doi.org/10.21203/rs.3.rs-778117/v1

License: (c) (i) This work is licensed under a Creative Commons Attribution 4.0 International License. Read Full License 


\section{Abstract}

Background: Human immunodeficiency virus type 1 (HIV-1) causes impairment of T and B cell responses, which begins during the acute phase of infection and is not completely restored by antiretroviral treatment. Regulatory $T$ cell (Tregs) can improve overall disease outcome by controlling chronic inflammation, but may also suppress beneficial HIV-1 specific immune responses. We analyzed immune alterations, including Tregs, and their clinical significance in Mozambican people living chronically with HIV-1 (PLWH-C).

Results: In PLWH-C, the proportion of total Tregs was positively correlated with the proportion of IL- $2^{+}$CD 4 T cells $(r=0.647 ; p=0.032)$ and IL- $2^{+}{ }^{+} F N{ }^{+} C D 8$ T cells $(r=0.551 ; p=0.014)$, while the Helios ${ }^{+}$Tregs correlated inversely with levels of IL-2 ${ }^{+} \mathrm{CD} 8 \mathrm{~T}$ cells $(r=-0.541 ; p=0.017)$. Overall, PLWH-C, with $(82 \%)$ or without virologic suppression (64\%), were seronegative for at least HIV-1 p31, gp160 or p24, and the breadth of antibody responses was positively correlated with proportions of $C D 38^{+} H L A-D R^{+} C D 8 T$ cells $(r=0.620$; $p=0.012)$, viral load $(r=0.452 ; p=0.040)$ and inversely CD4 T cells count $(r=-0.481 ; p=0.027)$. Analysis of all individuals living HIV-1 showed that the breadth of HIV-1 antibody responses was inversely correlated with the proportion of Helios ${ }^{+}$Tregs $(r=-0.45 ; p=0.02)$.

Conclusion: Among Mozambican patients living with HIV-1, seronegativity to some HIV-1 proteins is common mostly in virologically suppressed individuals. Low diversity of HIV-specific antibodies is correlated to indicators of disease control during ART. Elevation in the proportion of Helios ${ }^{+}$Tregs is related to a reduction of CD8 T expressing intracellular IL-2 but may contribute to impairment of $B$ cell function.

\section{Introduction}

Human Immunodeficiency Virus type 1 (HIV-1) infection causes generalized immunodeficiency which is characterized by a profound depletion of CD4 T cells, impairment of B and T cells function, and systemic immune activation which persists during the chronic phase of the disease $[1,2]$. During the acute phase of HIV-1 infection, disruption of the gut mucosa, associated with depletion of CD4 T cells at the gut epithelium, is proposed to result in massive microbial translocation into the blood $[3,4]$. This culminates with activation of innate and adaptive mediators of the immune response, and a persistent inflammatory environment and immune exhaustion [5, 6].

Chronic immune activation induced by HIV-1 infection also alters the lymphoid tissue architecture leading to impairment of immune reconstitution [5]. Additionally, there is a loss of HIV-specific memory B cells, probably associated with loss of HIV-specific CD4 T cells, which is not restored by antiretroviral therapy (ART) $[7,8]$. It has also been postulated that effective virologic control with ART may lead to loss of antiHIV antibodies as a consequence of reduced antigenic stimulation [9]. However, a report of eighty-four people living chronically with HIV-1 (PLWH-C) and undetectable viral loads for longer than five years under ART showed that seroreversion measured using HIV-1 Western blot is a very rare event occurring in 
only one patient [10]. Thus, the significance of reduced HIV-1 specific antibody reactivity on clinical diagnostic assays, particularly in PLCWH remains to be determined.

Regulatory T cells (Tregs) are a subset of CD4 T cells with the potential to suppress T and B cell functions by a vast array of mechanisms such as IL-2 deprivation and secretion of suppressor mediators [11]. Tregs are identified by expression of various markers including transcription factor Foxp3 and the a chain of IL-2 receptor (CD25) [11]. Other markers, including Helios and CD45RA are associated with Tregs function [12-14]. Expression of the transcription factor Helios identifies stable and highly suppressive Tregs $[15,16]$. Deletion of Helios on Tregs in mice leads to progressive systemic immune activation, enhanced germinal center formation and conversion to effector $T$ cells $[15,17]$. We previously reported that in Mozambican people living early with HIV-1, at elevated proportion of Helios expressing Tregs, lower levels of HIV-1 viral replication and recovery of CD 4 T cells absolute counts were observed [18]. This study aimed to assess the alterations of the T cells immune profile and circulating HIV- 1 specific antibodies in PLWH-C and their correlation with expression of the suppressive marker Helios on Tregs.

\section{Methods}

\subsection{Study participants}

Forty participants enrolled in RV363 were included in this study. RV363 was a prospective study that assessed HIV-1 incidence, retention rate and willingness of adults to participate in future HIV vaccine trials in Mozambique, as previously described [19]. People living without HIV-1 (PLWOH), classified as high risk for HIV-1 acquisition, were screened every 3 months for HIV-1 antibodies over a 2-years period. For this study, 7 participants were living early with HIV-1 (PLWH-E), (within 3 months) and 24 participants were PLWH-C (defined as living with HIV for more than 12 months). Twelve of PLWH-C had detectable viral load whereas the other 12 had viral suppression (VS) for at least one year. Nine PLWOH were included in the study as control group. All study participants provided written informed consent to participate in this study.

\subsection{HIV diagnosis, profile of reactivity to HIV-1 proteins, CD4 counts and HIV-1 viral load testing}

HIV-1 diagnosis was performed on fresh venous whole blood samples following the Mozambican national algorithm for HIV testing, which consists of two sequential rapid immunochromatographic tests for detection of anti-HIV-1/2 antibodies. The screening was first performed using the Alere Determine ${ }^{m}$ HIV-1/2 (Abbott, USA) rapid test. Participants that the specimens did not react to the test were diagnosed as non-living with HIV-1. Reactive specimens were confirmed by a second rapid test (Uni-Gold ${ }^{\circledR} H I V$, Trinity Biotech PLC, Ireland). Discordant results were resolved by a fourth-generation ELISA (Genscreen Ultra HIV Ag-Ab (BioRad, France) kit, using stored plasma or serum. The antibody reactivity pattern for HIV-1 - Env (gp160 and gp41), Gag (p24) and Pol (p31) proteins was analyzed on stored serum samples from all individuals living with HIV-1, using the Geenius HIV 1/2 Confirmatory Assay (BioRad, France). 
CD4 T cells were enumerated from fresh EDTA-whole blood by four-color flow cytometry (FACS Calibur, Becton Dickinson [BD], USA). Plasma HIV-1 viral load was measured using the COBAS AmpliPrep/COBAS TaqMan HIV-1 Test, v2 (Roche, USA).

\subsection{Peripheral blood mononuclear cells isolation}

Peripheral blood mononuclear cells (PBMC) were isolated within 8 hours of phlebotomy from heparin anti-coagulated blood using Ficoll-Paque Plus (GE Healthcare, Sweden) and Leucosep tubes (Greiner BioOne, German). PBMC were cryopreserved in 10\% dimethyl sulfoxide (DMSO) $+90 \%$ fetal calf serum (FCS) in liquid nitrogen at or below $-140^{\circ} \mathrm{C}$.

\subsection{In vitro stimulation of peripheral blood mononuclear cells}

PBMC were thawed in a water bath at $37^{\circ} \mathrm{C}$ and washed twice in complete RPMI medium supplemented with $20 \%$ FCS followed by $10 \%$ FCS. Viable cells were counted using a Nucleocounter (Chemometec, Denmark). PBMC were rested overnight in complete RPMI medium supplemented with 10\% FCS (R10 medium) at $37^{\circ} \mathrm{C} / 7.5 \%$ CO2. Subsequently, 500,000 PBMC, suspended in $100 \mu \mathrm{L}$ of R10 medium, were mixed with monensin, brefeldin A, and co-stimulatory monoclonal antibodies against CD28 $(1 \mu \mathrm{g} / \mathrm{mL})$ and CD49d $(1 \mu \mathrm{g} / \mathrm{mL})$ diluted in R10 medium. PBMC's were then stimulated with $50 \mu \mathrm{L}$ of Staphylococcus enterotoxin $A$ and $B(S E A B,(1 \mu \mathrm{g} / \mathrm{mL})$ ) or were maintained with $0.5 \%$ of DMSO in R10 medium (negative control). Cell suspensions were incubated for six hours at $37^{\circ} \mathrm{C} / 7.5 \% \mathrm{CO} 2$ and subsequently stored overnight at $2-8^{\circ} \mathrm{C}$ prior to analysis. PBMC were unstimulated for determination of $\mathrm{T}$ cells activation markers.

\subsection{Immunophenotyping}

Following overnight exposure to antigen or medium, PBMC were treated with 20mM EDTA and incubated for $15 \mathrm{~min}$ in the dark at room temperature. Cells were then washed with phosphate buffered saline (PBS) prior to staining with $50 \mu \mathrm{L}$ of viability dye (fixable viability stain (FVS) $510, \mathrm{BD}$, USA). PBMC's were kept in the dark at room temperature, for another 15 minutes, then washed twice with 5\% FCS in PBS. After washing, PBMC were stained with monoclonal antibodies cocktails for identification of cell surface markers. Cells used for intracellular staining of cytokines and FoxP3 were fixed with $200 \mu \mathrm{L}$ of $1 \mathrm{X}$ Human FoxP3 Buffer A (BD, USA) for 10 min at $4^{\circ} \mathrm{C}$. PBMC were then washed with diluted BD Perm/Wash buffer (BD, USA) and permeabilized with Human FoxP3 Buffer $\mathrm{C} 1 \mathrm{X}$ for 30 minutes in the dark at room temperature. Prior to staining of PBMC, for detection of intracellular marker, cells were washed twice with BD Perm/Wash buffer (BD, USA). The combinations of monoclonal antibodies for intracellular staining were added to the cells and incubated at $2-8^{\circ} \mathrm{C}$. After 30 minutes, cells were washed twice with Perm/Wash buffer (BD, USA) and finally treated with $200 \mu \mathrm{L}$ of $1 \mathrm{X}$ BD CellFix (BD, USA). The following combinations of monoclonal antibodies and the viability dye, all from BD, USA, were used: (1) Activation Markers: $\mathrm{CD}^{\mathrm{FITC}} / \mathrm{HLA}-\mathrm{DR}{ }^{\mathrm{PE}-\mathrm{Cy} 7} / \mathrm{CD} 38^{\mathrm{APC}} / \mathrm{CD} 8^{\mathrm{APC}-\mathrm{H7}} / \mathrm{CD} 4^{\mathrm{V} 450} /$ FVS510; (2) Tregs: CD $4^{\mathrm{FITC}}$ / 
FoxP3 ${ }^{\mathrm{PE}} / \mathrm{CD} 45 \mathrm{RA}$ PerCP - Cy5.5/ CD25 ${ }^{\mathrm{PE}-\mathrm{Cy} 7} / \mathrm{Helios}^{\mathrm{APC}} / \mathrm{CD}^{\mathrm{APC}-\mathrm{H7}} / \mathrm{IFNy}$ 450/ FVS510 and (3) Intracellular cytokine staining: $\mathrm{CD}^{\mathrm{FITC}} / \mathrm{IFNY} \mathrm{PE}^{\mathrm{PCy}} / \mathrm{IL}^{\mathrm{APC}} / \mathrm{CD} 8^{\mathrm{APC}-\mathrm{H} 7} / \mathrm{CD} 4 \mathrm{V450} / \mathrm{FVS} 510$.

After 30 minutes, samples were analyzed on a FACSCanto II (BD, USA). A minimum of 100,000 events were acquired using Diva software version 8 (BD, USA). For quality control purposes, the BD Cytometer Setup \& Tracking (CST) beads and BD Comp beads were used to ensure consistency of results over time and for compensation, respectively. The post-acquisition analyses, including compensation, were performed using FlowJo software, version 10 (FlowJo LLC, USA). Gating strategy for definition of Tregs is shown in Fig. 1a-h.

\subsection{Statistical analysis}

Statistical analyses were performed using GraphPad Prism version 6.0h (USA). The Mann-Whitney test was used to test heterogeneity among different groups. Correlations between two variables were performed by the Spearman Rank correlation. Differences or correlations with $p$-values less than 0.05 were considered statistically significant.

\section{Results}

\subsection{Study participant characteristics}

An overview of the study participants is shown in Table 1. Eight PLWH-C without VS were not receiving ART at the time of PBMC collection (median HIV-1 viral load was $\log _{10} 4.4$ copies $/ \mathrm{mL}$ (4.1-4.9)). Two PLWH-C on ART and with viral load of less than 20 viral RNA copies/mL were included in the group of individuals with VS. The absolute number of CD4 T cells was lower in PLWH-C with or without VS compared to PLWOH study group. 
Table 1

Characteristics of study participant groups

\begin{tabular}{|lllll|}
\hline & $\begin{array}{l}\text { PLWH-C } \\
\text { without VS }\end{array}$ & PLWH-C with VS & PLWH-E & PLWOH \\
\hline $\mathrm{N}$ & 12 & 12 & 7 & 9 \\
\hline Age (years), median (IQR) & $23(21-28)$ & $22(18-27)$ & $22(21-30)$ & $20(19-24)$ \\
\hline Sex (Female/Male) & $7 / 5$ & $5 / 7$ & $4 / 3$ & $5 / 4$ \\
\hline \#CD4 (cells/ $\mu \mathrm{L})$, Median (IQR) & $\begin{array}{l}477(324- \\
640)^{*}\end{array}$ & $525(462-628)^{*}$ & $604(401-$ & $763(740-$ \\
\hline HIV-1 viral load (median $\log _{10}$ & $4.4(4.1-4.9)$ & Undetectable or & $4.4(3.1-$ & N/A \\
(IQR)) & $<1.3$ & $5.6)$ & \\
\hline $\begin{array}{l}\text { Time after first positive result } \\
\text { (months), }\end{array}$ & $>12$ & $>12$ & 3 & N/A \\
\hline \# Participants on ART (N) & 4 & 12 & 0 & N/A \\
\hline
\end{tabular}

IQR - interquartile range. M/F - male/female. N/A - not applicable. ART - Antiretroviral therapy. VS viral suppression.

${ }^{*} \mathrm{p}<0.05$ compared to HIV negative group.

\subsection{Individuals living with chronic infection with virologic control present decreased breadth of HIV-1 antibodies and lower levels of activated T cells}

We observed seronegativity to tested HIV-1 proteins in PLWH-C as shown in Table 2. All participants reacted to gp41. Overall, 28/29 (97\%) participants reacted to gp160, 14/29 (48\%) reacted to p31 and $19 / 29(66 \%)$ reacted to $p 24$. The proportions of individual with absence of reactivity to at least one protein was $81.8 \%$ for PLWH-C with VS but $63.6 \%$ for PLWH-C without VS.

We evaluated co-expression of activation markers CD38 and HLA-DR in unstimulated CD4 and CD8 T cells and intracellular expression of IL-2 and IFNy after stimulation with SEAB. Overall, the level of coexpression of HLA-DR and CD38 on CD4 and CD8 T cells was higher in PLWH-C compared to PLWOH ( $p=$ 0.005 and $p=0.019$, respectively) (Fig. 2a and 2b). However, after stratification in two groups - based on viral suppression status, we observed that this difference remained significant only in PLWH-C without VS for both activated CD 4 and CD8 T cells ( $p=0.003$ and $p<0.001$, respectively) (Fig. $2 c$ and $2 d)$. As expected the frequency of co-expression of activation markers on CD8 T cells correlated with HIV-1 viral load $(r=0.711 ; p<0.001)$

When evaluating people living with HIV without VS, including PLWH-E, the correlation between the frequency of co-expression of activation markers on CD8 T cells with HIV-1 viral loads was not observed 
$(r=0.348, p=0.157)$ (Fig. 2e). However we found a correlation between CD8 T cells expressing intracellular IFNy with viral loads $(r=0.656, p=0.007)$ and CD4 T cell counts $(r=0.665, p=0.006)(F i g .2 f$ and $2 \mathrm{~g}$ ).

\subsection{Individuals living chronically with HIV-1 the breadth of HIV-1 antibody responses correlates with co-expression of activation markers on CD8 T cells, viral replication and absolute CD4 T cell counts}

In PLWH-E, the frequency of seroreactivity to HIV-1 proteins correlated inversely with activation of CD8 T cells and positively with CD $4 \mathrm{~T}$ cell absolute counts [18]. Here we found that for participants with chronic infection, the breadth of antibody response to HIV-1 proteins correlated positively with the levels of coexpression of HLA-DR and CD38 on CD8 T cells $(r=0.620 ; p=0.012)$, HIV- 1 viral load $(r=0.452 ; p=0.040)$ and inversely with CD4 T cell counts $(r=-0.481 ; p=0.027$ ) (Fig. 3a, 3b and 3c). Co-expression of HLA-DR and CD38 on CD4 T cells did not correlate with the breadth of antibody response to HIV-1 proteins ( $\mathrm{r}=$ $0.269 ; p=0.328$.

Table 2

Summary of the HIV-1 specific antibody profiles to four HIV-1 proteins in individuals living with HIV-1

\begin{tabular}{|lllll|}
\hline & PLWH-C without & $\begin{array}{l}\text { PLWH-C with } \\
\text { VS }\end{array}$ & $\begin{array}{l}\text { PLWH- } \\
\text { ES }\end{array}$ & Total \\
\hline N & 11 & 11 & 7 & 29 \\
\hline Reactivity to HIV-1 p31 (\%) & $63.6 \%$ & $27.3 \%$ & $28.0 \%$ & $48.3 \%$ \\
\hline Reactivity to HIV-1 gp160 (\%) & $100 \%$ & $90.9 \%$ & $100 \%$ & $96.6 \%$ \\
\hline Reactivity to HIV-1 p24 (\%) & $72.7 \%$ & $45.5 \%$ & $85.7 \%$ & $65.5 \%$ \\
\hline Reactivity to HIV-1 gp41 (\%) & $100 \%$ & $100 \%$ & $100 \%$ & $100 \%$ \\
\hline $\begin{array}{l}\text { Non-reactive at least to one of the tested } \\
\text { proteins (\%) }\end{array}$ & $63.6 \%$ & $81.8 \%$ & $57 \%$ & $69 \%$ \\
\hline
\end{tabular}

\subsection{The frequency of Helios expressing Tregs, but not total Tregs, correlate with decreased proportions of CD8 T cells producing IL-2 in people living chronically with HIV and decreased frequency of antibodies reactive to HIV-1 proteins}


Persistent inflammation is considered the main promoter of immunologic failure in PLWH [5]. We measured the proportions of unstimulated Tregs and effector CD4 and CD8 T cells expressing IL-2 and IFNy after PBMC stimulation with SEAB. The proportions of Tregs in PLWH-C did not differ significantly from those observed in PLWOH $(p=0.213)$. The Tregs proportions in PLWH-C was correlated with the proportion of CD4 T cells expressing intracellular IL-2 $(r=0.467 ; p=0.033)$ or CD8 T cells expressing IL-2 in combination with IFNy $(r=0.551 ; p=0.014)$ (Fig. $4 a$ and $4 b)$. When assessing all study participants living with HIV-1 but without VS, including PLWH-E, the same correlation between the proportions of Tregs and CD8 T cells, producing IL-2 combined with IFNy was also observed $(r=0.574 ; p=0.031)$. Furthermore, Tregs proportions were also correlated with proportions of CD8 T cells, producing IL-2 $(r=0.515, p=$ $0.043)$ and IFNY $(r=0.512, p=0.045)$ alone and with CD4 T cells expressing activation markers $(r=0.549$, $\mathrm{p}=0.018)$.

When evaluating all study participants living with HIV-1 but without VS, including PLWH-E we found a positive correlation between Tregs with the viral loads $(r=0.505, p=0.033)$ and trended to correlate inversely with absolute CD 4 counts $(r=-0.465 ; p=0.052)$ (Fig. 4c and $4 d)$. However, in PLWH-C but with VS, we found a positive correlation between Tregs and absolute CD4 $T$ cells counts $(r=0.719, p=0.034)$ (Fig. 4e).

Regarding the Tregs expressing the transcription factor Helios we found an inverse correlation with the proportions of CD8 T cells expressing IL-2 ( $r=-0.541 ; p=0.017)$ (Fig. $5 a)$. Moreover, when grouping all individuals living with HIV-1, including PLWH-E, we found that the proportion of Tregs expressing Helios correlated inversely with the breadth of antibodies response to HIV-1 proteins $(r=-0.458 ; p=0.024)$ (Fig. $5 b)$.

\section{Discussion}

Early studies of HIV-specific CD8 T cells response in individuals living with HIV-1 showed an inverse correlation between the early emergence of CD8-specific responses and plasmatic viral levels [20]. However, the analysis of total HIV-, Env- and Nef-specific CD8 T cells producing IFNy showed a positive correlation with viral load [21]. Further studies suggested that CD8 T cell response, specifically Gagspecific response, may have different roles according to the stage of the infection [22]. In this study, we also measured the levels of intracellular cytokines IL-2 and IFNY in CD8 T cells stimulated with SEAB and found that the proportions of those expressing IFNY correlated directly with HIV-1 viral load and inversely with absolute CD4 counts. Our results suggest that levels of IFNy expression on CD8 T cells reflects T-cell activation driven by HIV-1 viral replication.

As observed in a previous report of early HIV infection [18] the proportion and absolute count of Tregs remained unaltered in our cohort of PLWH-C relative to PLWOH, in spite of decreased frequencies and absolute counts of total CD4 T cells. This could mean that during HIV-1 infection, Tregs might be preserved within the total CD4 T cells compartment. Indeed, studies have reported expansion of Tregs within CD4 T cells in people living with HIV-1, probably as a consequence of increased immune activation 
[23]. Furthermore, the unaltered proportion of Tregs could also be due to increased migration of Tregs from other tissues to the peripheral blood, as suggested by our previous results of increased expression of CXCR3 and CCR5 on Tregs [18], or a combination of factors.

Contrary to what we observed in people living with HIV without VS, including PLWH-E, the relative frequency of Tregs, in PLWH-C and with suppressed viral replication, correlated with increased absolute CD4 T cell counts. This observation suggests that in conditions of VS, Tregs expand in parallel with the recovery of CD4 T cell counts. However, in spite of this apparent immune restoration in PLWH-C and suppressed viral replication, it seems that Tregs are not able to control immune activation, since higher levels of CD8 T cell producing pro-inflammatory cytokines are observed despite high levels of total Tregs in these individuals.

After observing that high frequency of IFNy expression in CD8 T cells in people living with HIV-1 without VS correlated with increased viral load, decreased CD 4 counts and total Tregs, we assessed how the profile of these CD8 T cells correlated with expression of the transcription factor Helios in Tregs, which represents Tregs with a stable suppressive function. Although we did not find a significant correlation when evaluating IFNy producing CD8 T cells, we found that in individuals with increased frequencies of Tregs expressing the transcription factor Helios, lower proportions of IL-2 expressing CD8 T cells were observed. These observations suggest that in chronic HIV-1 infection, Tregs expressing Helios may have the potential to suppress systemic inflammation. Suppression of activated or pro-inflammatory CD8 T cells could be one of the contributions of Helios ${ }^{+}$Tregs controlling the deleterious effects of HIV-1 infection. This is aligned with the inverse correlation that we previously observed between Helios ${ }^{+}$Tregs and plasmatic viral loads [18].

Here, we also found that decreased breadth of reactivity to HIV-1 proteins is a frequent event in subjects living with chronic HIV-1, more evident in those on ART, and is correlated with immune regulation and virologic control. During HIV infection, Tregs are thought to be important in limiting the levels of systemic immune activation but Tregs can also suppress the development of HIV-specific protective $T$ and $B$ cell immune responses $[23,24]$. When characterizing the antibody profile against different HIV-1 antigens, we observed that irrespective of infection for more than one year, participants living chronically with HIV-1 tended to show incomplete HIV-1 specific antibody profiles. This was more pronounced in virologically suppressed ART-treated individuals living chronically with HIV, with $80 \%$ presenting seronegativity to at least one of the tested HIV-1 proteins. However, a previous study reported seroreversion of HIV-1 antibodies as a rare event in PLWH-C [10]. These contradictions might be in part due to the assays and HIV target proteins used for each study. In that study the authors targeted all HIV-1 proteins, with remarks to gp160, gp41, p24 and gp120 and used Western blot assay while in our study we only targeted gp160, gp41, p24 and p31 and used a recombinant protein assay. Seroreversion of antibodies targeting HIV-1 proteins has been described in patients living with HIV-1 treated shortly after primary infection [9] [2527]. However, our study was cross-sectional and time of ART initiation was unknown so seroreversion could not be assessed. Seroreversion on some HIV-1 antibodies assays has also been reported in late stage disease when $C D 4^{+} T$ cell counts are extremely low $[9,28]$. The cause of HIV-1 antibody loss has 
been attributed to reduced antigenic stimulation in individuals with effective virologic control by ART [9]. In this regard, we observed that absence of seroreactivity to HIV-1 proteins correlated with virologic control, decreased expression of activation markers on CD8 T cells and recovery of CD4 T cell counts. Thus, we suggest that in chronic infection, while increased production of IL-2 and IFNY is associated with laboratory indicators of disease progression seronegativity to some HIV-1 proteins is associated with indicators of disease recovery.

With regard to the impact of Tregs on decreased seroreactivity to HIV-1, it has been suggested that accumulation of Tregs that secrete TGF- $\beta 1$ in the lymph nodes attempting to control persistent inflammation are the major contributors of collagen deposition that characterizes chronic infection [1]. Collagen deposition in lymph nodes is associated with loss of follicular dendritic cells and fibroblastic reticular cells during the course of HIV-1/SIV infection [1]. We found that individuals with decreased seroreactivity to HIV-1 proteins had higher proportions of Tregs expressing Helios. We hypothesize that the relative frequency of Tregs expressing Helios, despite contributing to control of chronic inflammation, contribute to impaired B cell function and the ensuing seronegativity.

Altogether, our study results suggest that decreased seroreactivity to HIV-1 is frequent in ART-treated virologically suppressed individuals living chronically with HIV-1 and could be a reflection of decreased stimulation of HIV-1 specific B due to absence of viral antigen(s). Furthermore, our results raise the hypothesis that elevation of Tregs with higher suppressive potential, but not total Tregs, may have a beneficial impact controlling the levels of inflammation and viral loads but may compromise B-cell function. Further studies are needed to sustain that hypothesis.

The main limitations of this study are: (1) the small sample size and lack of same data for some participants due to failures during the experiments (2) the lack of information regarding the ART regimen, and the time from initiation of ART, (3) the duration of HIV-1 infection in participants living chronically with HIV-1, (4) assessment of a limited number of inflammatory markers and (5) the lack of assessment of Tregs subsets function.

\section{Abbreviations}

ART: Antiretroviral therapy

CNBS: Mozambican National Committee on Bioethics in Health (Comité Nacional de Biotética em Saúde de Moçambique);

DMSO: Dimethyl sulfoxide;

FCS: Fetal calf serum;

FVS 510: Fixable viability stain 510;

Foxp3: Transcription factor forkhead box P3; 
HIV-1: Human Immunodeficiency Virus type 1;

PBMC: Peripheral blood mononuclear cells;

PBS: Phosphate buffered saline;

Tregs: Regulatory $\mathrm{CD} 4^{+} \mathrm{T}$ cells;

PLWH-C. people living chronically with HIV-1

PLWH-E: people living early with HIV-1

PLWOH. people living without HIV-1

VS: virologic suppression.

\section{Declarations}

\section{Ethics approval and consent to participate}

The regulatory authorities in Mozambique, United States of America and Belgium, the Mozambican National Ethics Committee (Comité Nacional de Bioética em Saúde) (282/CNBS/13), the Walter Reed Army Institute of Research (WRAIR) Institutional Review Board (WRAIR \#2250), and the Medical Ethics Committee of the Institute of Tropical Medicine, Belgium (1065/15) approved this study. All participants consented to participate in this study.

\section{Consent for publication}

Not applicable

\section{Availability of data and materials}

The datasets used and analyzed during the current study are available from the corresponding author on reasonable request.

\section{Competing interests}

The authors have declared that no competing interests exist.

\section{Funding}

This work was supported by a cooperative agreement (W81XWH-07-2-0067) between the Henry M. Jackson Foundation for the Advancement of Military Medicine, Inc., and the U.S. Department of Defense (DOD). This research was funded, in part, by the U.S. National Institute of Allergy and Infectious Diseases. Some funds were also received from the Flanders Department of Foreign Affairs of the Flemish 
Government (BICMINS). The funding sources had no involvement in: study design, data collection, analysis, interpretation of data, writing the manuscript nor in decision to submission of the article for publication. Material has been reviewed by the Walter Reed Army Institute of Research. There is no objection to its presentation and/or publication. The opinions or assertions contained herein are the private views of the author, and are not to be construed as official, or as reflecting true views of the Department of the Army or the Department of Defense. The investigators have adhered to the policies for protection of human subjects as prescribed in AR 70-25.

\section{Authors' contributions}

RMC, CM, MR, VM and VC performed the experiments. RMC, ME, IM, NB, CSP and MI participated in study coordination. CSP provided supervision, and CSP and MI provided project administration. RMC performed the data analysis, interpretation and drafted the manuscript. RMC, IJ and LK designed the study, conceived the experiments, and wrote the manuscript. All authors reviewed the manuscript, provided feedback, and approved of the manuscript in its final form.

\section{Acknowledgements}

We are thankful to all participants who made this work possible. Special thanks goes to Mark de Souza for his robust comments and reviews of this manuscript and the institutions that supported this work including the Instituto Nacional de Saúde (INS), Polana Caniço Health Research and Training Center (CISPOC), Henry M. Jackson Foundation for the Advancement of Military Medicine (HJF), HJF Medical Research International (HJFMRI), US Military HIV Research Program (MHRP), and the Walter Reed Army Institute of Research (WRAIR). The authors are also thankful to the study participants who made this work possible. The RV363 Study Group includes: Julie Ake, Khelvon De Araujo, Nilesh Bhatt, Igor Capitine, Raquel Matavele Chissumba, Alberto Machaze, Eduardo Namalamgo, Celso Castiano, Emelva Manhiça, Mirna Mutombene, Ducília Matimbe, Onélia Guiliche, Vania Mapossa, Vania Monteiro, Nilzio Cavele, Trevor Crowell, Leigh Anne Eller, Zebiba Hassen, Michelle Imbach, Luis Inhambizo, Ilesh V. Jani, Qun Li, Ivalda Macicame, Ferrao Mandlate, Carmélia Massingue, Mark Milazzo, Vanessa Monteiro, Chiaka Nwoga, Christina S. Polyak, Patrícia Ramgi, Merlin L. Robb, Steve Schech, Gail Smith, Edith M. Swann, Edna Viegas, and Adam Yates.

\section{References}

1. Dimopoulos, Y., E. Moysi, and C. Petrovas, The Lymph Node in HIV Pathogenesis. Curr HIV/AIDS Rep, 2017. 14(4): p. 133-140.

2. Hazenberg, M.D., et al., Persistent immune activation in HIV-1 infection is associated with progression to AIDS. AIDS, 2003. 17(13): p. 1881-8.

3. Dinh, D.M., et al., Intestinal microbiota, microbial translocation, and systemic inflammation in chronic HIV infection. J Infect Dis, 2015. 211(1): p. 19-27. 
4. Marchetti, G., C. Tincati, and G. Silvestri, Microbial translocation in the pathogenesis of HIV infection and AIDS. Clin Microbiol Rev, 2013. 26(1): p. 2-18.

5. Nasi, M., et al., Persistent inflammation in HIV infection: established concepts, new perspectives. Immunol Lett, 2014. 161(2): p. 184-8.

6. Rychert, J., et al., Detection of HIV gp120 in plasma during early HIV infection is associated with increased proinflammatory and immunoregulatory cytokines. AIDS Res Hum Retroviruses, 2010. 26(10): p. 1139-45.

7. Bussmann, B.M., et al., Loss of HIV-specific memory B-cells as a potential mechanism for the dysfunction of the humoral immune response against HIV. Virology, 2010. 397(1): p. 7-13.

8. Kassutto, S., M.N. Johnston, and E.S. Rosenberg, Incomplete HIV type 1 antibody evolution and seroreversion in acutely infected individuals treated with early antiretroviral therapy. Clin Infect Dis, 2005. 40(6): p. 868-73.

9. Hare, C.B., et al., Seroreversion in subjects receiving antiretroviral therapy during acute/early HIV infection. Clin Infect Dis, 2006. 42(5): p. 700-8.

10. Amor, A., et al., Seroreversion of HIV antibodies in patients with prolonged suppression of viraemia under HAART. AIDS, 2006. 20(10): p. 1460-2.

11. Sakaguchi, S., et al., Regulatory T cells and immune tolerance. Cell, 2008. 133(5): p. 775-87.

12. Daniel, V., et al., Interferon-gamma producing regulatory $T$ cells as a diagnostic and therapeutic tool in organ transplantation. Int Rev Immunol, 2014. 33(3): p. 195-211.

13. Getnet, D., et al., A role for the transcription factor Helios in human CD4(+)CD25(+) regulatory $T$ cells. Mol Immunol, 2010. 47(7-8): p. 1595-600.

14. Miyara, M., et al., Functional delineation and differentiation dynamics of human CD4+ T cells expressing the FoxP3 transcription factor. Immunity, 2009. 30(6): p. 899-911.

15. Sebastian, M., et al., Helios Controls a Limited Subset of Regulatory T Cell Functions. J Immunol, 2016. 196(1): p. 144-55.

16. Kim, H.J., et al., Stable inhibitory activity of regulatory $T$ cells requires the transcription factor Helios. Science, 2015. 350(6258): p. 334-9.

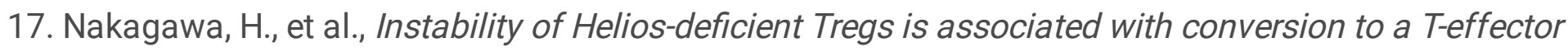
phenotype and enhanced antitumor immunity. Proc Natl Acad Sci U S A, 2016. 113(22): p. 6248-53.

18. Matavele Chissumba, R., et al., Helios + Regulatory $T$ cell frequencies are correlated with control of viral replication and recovery of absolute CD4 T cells counts in early HIV-1 infection. BMC Immunol, 2017. 18(1): p. 50.

19. Macicame, I., et al., HIV prevalence and risk behavior among male and female adults screened for enrolment into a vaccine preparedness study in Maputo, Mozambique. PLoS One, 2019. 14(9): p. e0221682.

20. Borrow, P., et al., Virus-specific CD8+ cytotoxic T-lymphocyte activity associated with control of viremia in primary human immunodeficiency virus type 1 infection. J Virol, 1994. 68(9): p. 6103-10. 
21. Betts, M.R., et al., Analysis of total human immunodeficiency virus (HIV)-specific CD4(+) and CD8(+) T-cell responses: relationship to viral load in untreated HIV infection. J Virol, 2001. 75(24): p. 1198391.

22. Jiao, Y., et al., Correlation between gag-specific CD8 T-cell responses, viral load, and CD4 count in HIV1 infection is dependent on disease status. J Acquir Immune Defic Syndr, 2006. 42(3): p. 263-8.

23. Chevalier, M.F. and L. Weiss, The split personality of regulatory T cells in HIV infection. Blood, 2013. 121(1): p. 29-37.

24. Jenabian, M.A., et al., Regulatory T cells in HIV infection: can immunotherapy regulate the regulator? Clin Dev Immunol, 2012. 2012: p. 908314.

25. Keating, S.M., et al., Brief Report: HIV Antibodies Decline During Antiretroviral Therapy but Remain Correlated With HIV DNA and HIV-Specific T-Cell Responses. J Acquir Immune Defic Syndr, 2019. 81(5): p. 594-599.

26. Keating, S.M., et al., HIV Antibody Level as a Marker of HIV Persistence and Low-Level Viral Replication. J Infect Dis, 2017. 216(1): p. 72-81.

27. Killian, M.S., et al., The effects of early antiretroviral therapy and its discontinuation on the HIVspecific antibody response. AIDS Res Hum Retroviruses, 2006. 22(7): p. 640-7.

28. Gutierrez, M., et al., Seroreversion in patients with end-stage HIV infection. Vox Sang, 1994. 67(2): p. 238-9.

\section{Figures}
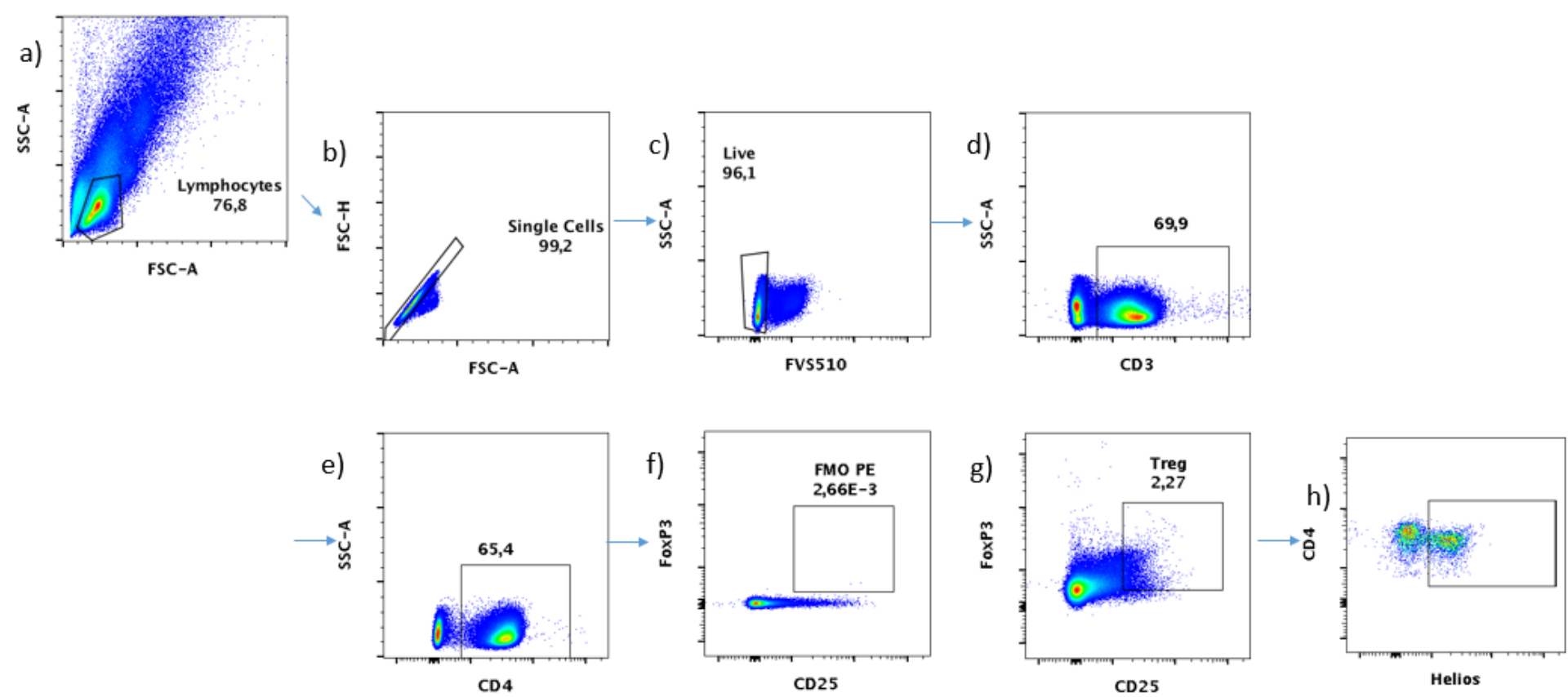

Figure 1 
Gating strategy for definition of Tregs by FlowJo. Gating strategy for: (a) identification of lymphocytes. (b) identification of singlets from lymphocytes. (c) definition of live cells from singlets. (d) identification of T cells from live cells. (e) identification of CD4 T cells from T cells. ( $f$ ) definition of the region for positivity of Tregs based on FMO control for FoxP3 in total CD4 T cells. (g) identification of Tregs as CD25HighFoxP3+ from total CD4 T cell. (h) definition of Helios positive cells from Tregs.
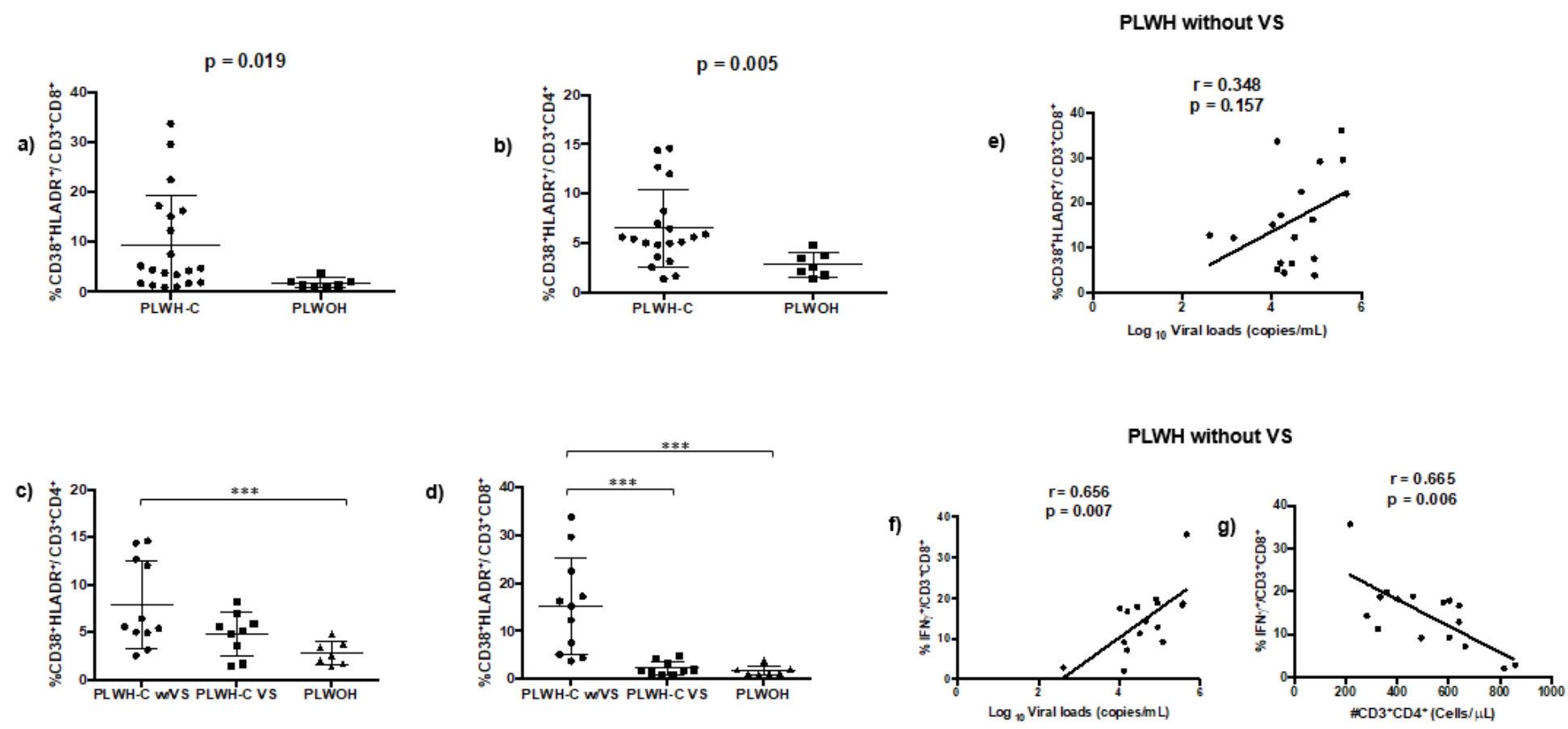

Figure 2

Activation of T cells and correlation with viral load. PBMC from PLWOH (7), PLWH-C (20) without VS (11) and with VS (9). PBMC were stained with monoclonal antibodies and analyzed by eight-color flow cytometer. $(a-d)$ Median and interquartile range for proportions of CD4 and CD8 T cells co-expressing CD38+HLA-DR+ in study groups. Each data point represents an individual. Unpaired Mann-Whitney test pvalues comparing medians from PLWOH and PLWH-C groups are indicated on each figure. (e) Correlation between the viral loads and proportion of CD8 T cells co-expressing CD38 and HLA-DR in PLWH without VS. Correlation between the proportion of CD8 T cells expressing IFNy with viral loads ( $f$ ) and CD4 T cell counts $(\mathrm{g})$ in PLWH without VS. Spearman's correlation $r$ and $p$-values are indicated $(* \star * p<0.0001)$. 
PLWH-C

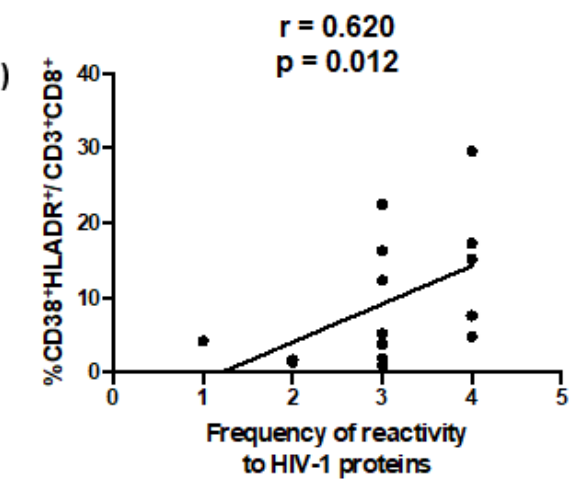

PLWH-C

b)

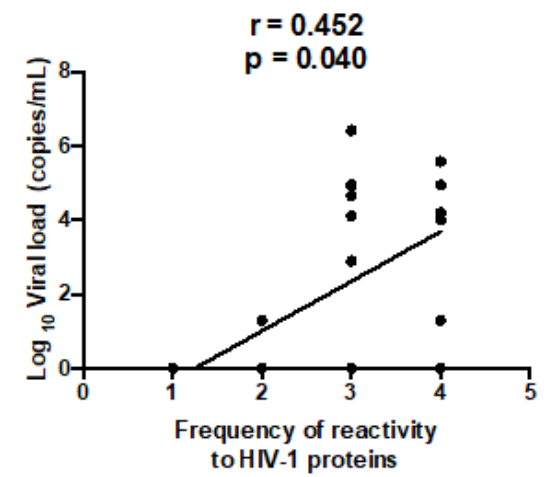

PLWH-C

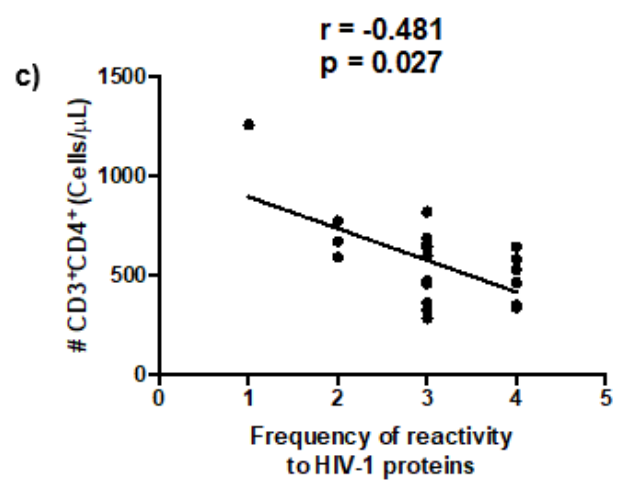

Figure 3

Correlations between frequency of antibodies reactive to HIV-1 proteins and immune restoration indicators. PBMC from PLWH-C $(n=20)$ were stained with monoclonal antibodies and analyzed by polychromatic flow cytometry. Absolute counts of CD4 T cells were determined using four-color flow cytometry. Serum antibody reactivity to different HIV-1 proteins. Correlations between the seroreactivity to HIV-1 proteins and (a) activation of CD8 T cells, (b) viral load and (c) absolute CD4 T cell counts in PLWHC. Each point corresponds to one individual. Spearman's correlation $r$ and $p$-values are indicated on each figure.

PLWH-C

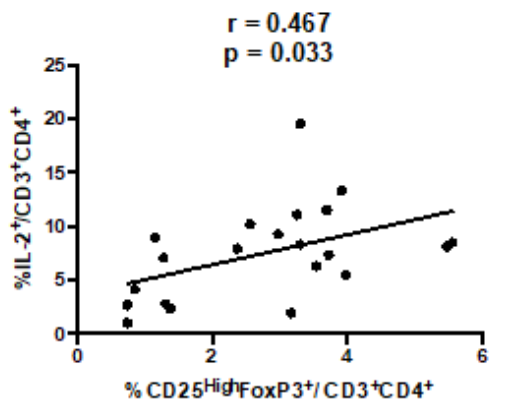

PLWH-C

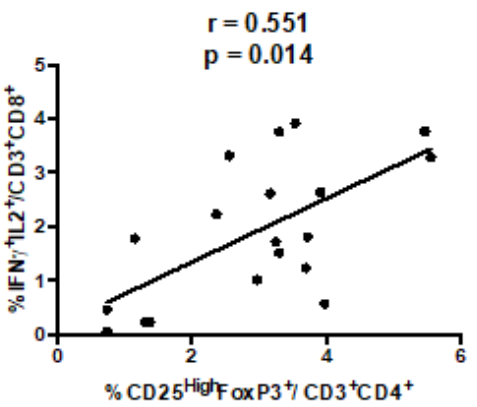

PLWH without VS

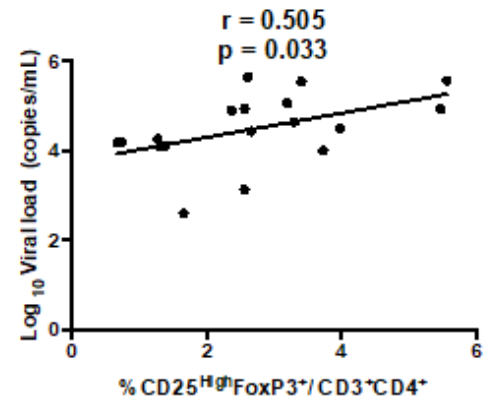

PLWH without VS

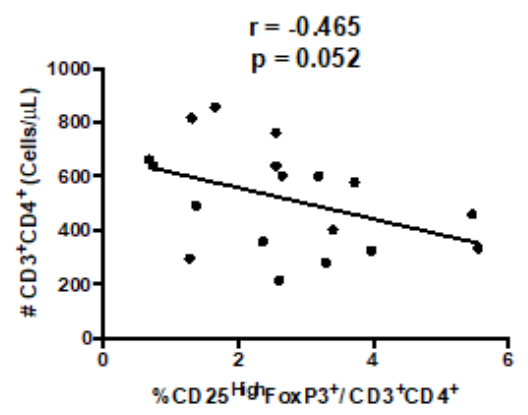

PLWH-C with VS

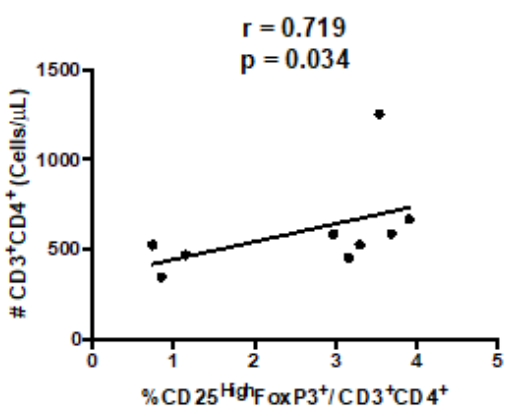




\section{Figure 4}

Correlations between Tregs and cytokine-producing CD4 and CD8 T cells, CD4 T cell absolute counts and viral loads. PBMC from PLWH-C were isolated and treated as described in methods section. PBMC were stained with monoclonal antibodies and analyzed by polychromatic flow cytometry. Absolute counts of CD4 T cells were determined using a four-color flow cytometer as described in the methods section. Correlation between the proportion of Tregs and (a) frequency of CD 4 T cells expressing IL-2 $(n=21)$ PLWH-C and (b) frequency of CD8 T cell expressing IL-2 combined with IFNY ( $n=19)$ PLWH-C. (c) Correlation between the proportions of Tregs within CD4 T cells and viral load $(n=18)$ and (d) CD4 T cells absolute counts in PLWH without VS $(n=18)$. (e) Correlation between the proportions of Tregs and CD4 T cells absolute counts in PLWH-C with VS $(n=9)$.

\section{PLWH-C}

a)

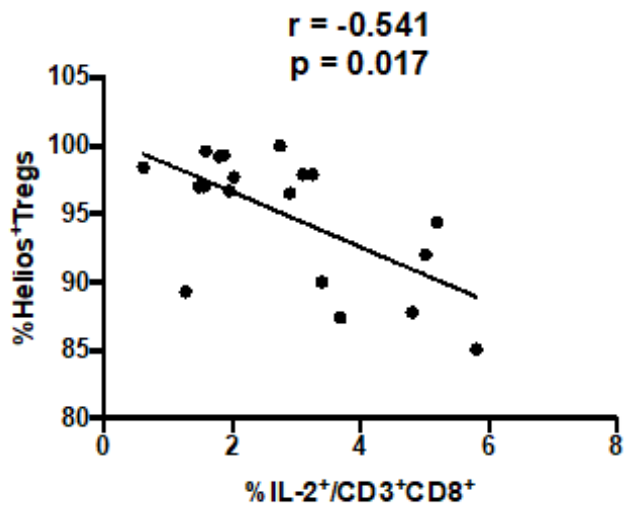

All PLWH

b)

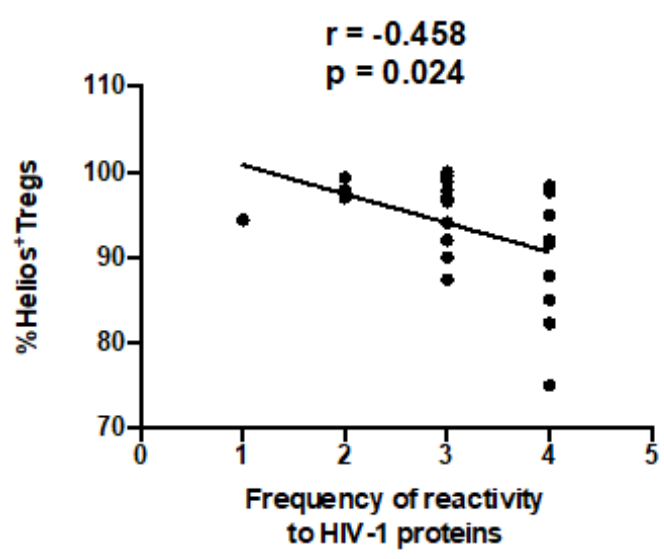

\section{Figure 5}

Correlation between proportions of Helios expressing Tregs (Helios+Tregs) and cytokine-producing CD8 T cells and seroreactivity to HIV-1 proteins. PBMC from PLWH were isolated and treated as described in the methods section. PBMC were stained with monoclonal antibodies and analyzed by polychromatic flow cytometry. Viral load and frequency of antibodies reactive to HIV-1 proteins were determined using plasma or serum samples as described in the methods section. Correlations between: (a) relative frequency of Helios expressing Tregs in PLWH-C and the proportion of CD8 T cells expressing IL-2 ( $n=19)$. (b) proportions of Helios expressing Tregs and reactivity to HIV-1 proteins for all PLWH $(n=24)$. Each data point corresponds to a single individual. Spearman's correlation $r$ and $p$-values are indicated on each figure. 